\title{
Robustly Computing Intersection Curves of Two Canal Surfaces with Quadric Decomposition
}

\author{
Jinyuan $\mathrm{Jia}^{1}$, Ajay Joneja ${ }^{2}$, and Kai Tang ${ }^{2}$ \\ ${ }^{1}$ Zhuhai College of Jilin University, \\ Jinwan District, Zhuhai, P. R. China, 519041 \\ csjyjialyahoo.com.cn \\ ${ }^{2}$ The Hong Kong University of Science and Technology, \\ Clear Water Bay, Kowloon, Hong Kong, P. R. China \\ Joneja@ust.hk, mektang@ust.hk
}

\begin{abstract}
This paper revisits the intersection problems of two canal surfaces with a new quadric decomposition we proposed for canal surfaces. It reduces computing intersection curves of two canal surfaces to computing intersection curves of two revolute quadrics. Furthermore, Bounding Cylinder Clipping is proposed for efficient intersection determination. Compared to the existing method, our method can $(i)$ run more robustly and efficiently; (ii) represent the final intersection curves as a piecewise closed-form RQIC; and (iii) give a simple shape analysis.
\end{abstract}

\section{Introduction}

Surface intersection is a fundamental issue in CAGD and geometric modeling. Robustness, accuracy and efficiency are used to evaluate surface intersection algorithms. Several important algorithms that were developed over few decades have been summarized in [16]. To design accurate, robust and efficient algorithms of computing intersection curves of two surfaces, even two special surfaces, e.g. quadrics, cyclides and canal surfaces still remains an open challenge. Although methods for general surface intersections can be applied to special surface intersection problems, they are inefficient. Considering that special surfaces usually have good geometric properties, it desirable to develop more efficient intersection algorithms for them. Therefore many papers have addressed specific intersection problems for CSG primitives, e.g. plane, sphere, cylinder, cone, quadric and tori $[1,2,3,5,13,14,17,18,19]$ and some potential geometric primitives e.g. cyclides, surfaces of revolution, ruled surfaces and ringed surfaces $[4,6,7,8,9,10,11,12,17]$.

Canal surfaces are one of important geometric primitives in solid modeling, VR, $\mathrm{CG}, \mathrm{CAD}$ and CAM. Examples of canal surfaces include natural quadrics, revolute quadrics, tori, Dupin cyclides, surfaces of revolution and pipe surfaces. Canal surfaces are very useful in representing long thin objects, for instance, pipes, poles, 3D fonts, brass instruments, internal body organs, and a variety of filleted surfaces. Therefore, it is essential to devise robust and efficient intersection algorithms for canal surfaces. 


\section{Related Works}

Subdivision is an important approach to solve surface intersection problems. Heo et al use circle decomposition to solve intersection problem of two canal surfaces in [6], which subdivides a canal surface into a dense set of characteristic circles, and reduces intersection problem of two canal surfaces to a zero-set searching problem of a bivariant function $f(u, v)=0$, that is much simpler than the original intersection problem. However, $(i)$ the numerical behavior of zero-set searching of $f(u, v)=0$ is both time and memory consuming at high precision; (ii) it outputs the intersection curves with a set of discrete sampling points, that is not easy to concatenate; and (iii) no shape analysis is performed on the intersection curves, e.g. loops and singularity.

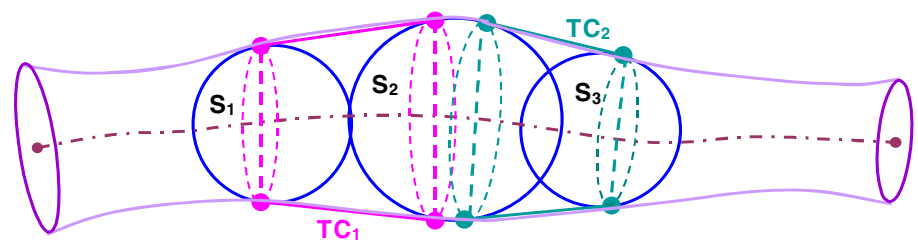

Cone-sphere decomposition

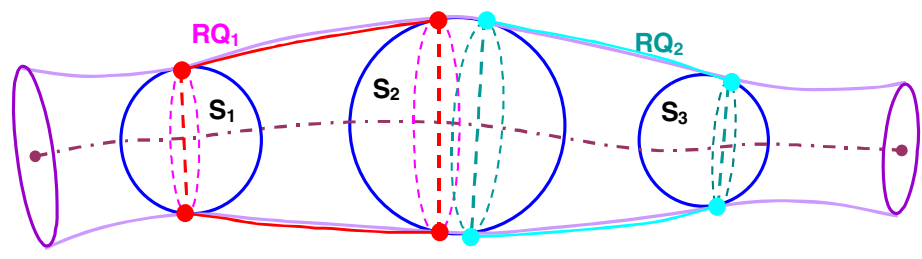

RQ-sphere decomposition

Fig. 1. Two quadric decomposition schemes of same canal surface: (a) Cone-sphere decomposition; (b) RQ-sphere decomposition

We revisit this problem by proposing more suitable subdivision scheme for canal surfaces. Except for circle decomposition, there are three other subdivision schemes for canal surfaces, cyclide [20], cone-sphere [15] and RQ-sphere [11]. The first one approximates a canal surface with a set of $G^{l}$ truncated cyclides [20]. The second one approximates a canal surface with a series of sampling spheres and associated tangential truncated cones (see Fig. 1(a)). However, the density of cone-spheres increases for good approximation quality at high precision and easily causes self-intersection of two neighboring truncated cones when the spine curve has high curvature. The last one approximates canal surfaces with a series of sampling spheres and associated tangential revolute quadrics (see Fig. 1(b)), instead of truncated cones. Apparently, not only less RQ-sphere pairs are required than cone-sphere scheme for same approximation quality, but the self-intersection problem can be avoided as well. In fact, cone-sphere is a special case of RQ-sphere, cyclide decomposition reduces canal/canal intersection computing to cyclide/cyclide intersection, that has to solve order 8 polynomial equation numerically. Both cone-sphere and $R Q$-sphere can reduce computing canal/canal intersection curves to computing $R Q / R Q$ intersection 
curves, that has closed-form solutions $[1,2,5,16,17,19,20]$. However, cone-sphere may yield incorrect intersection curve if it has self-intersection. So we decided to employ RQ-sphere decomposition to solve canal/canal intersection problem. In this paper, we propose a new conception, canal valid intersection intervals (CVII), a good hierarchical data structure, cylindrical bounding volume (BCT), and a new method, bounding cylinder clipping for efficient intersection determination in Section 3. The rough idea of canal/canal intersection algorithm is described in Section 4. Experimental examples given in Section 5 show the robustness and efficiency of our method. Conclusions and future work are presented in Section 6.

\section{Bounding Cylinder for Canal Surfaces}

Compared to traditional bounding volumes, bounding box and bounding sphere, bounding cylinder $\mathrm{BC}$ is chosen for canal/canal intersections because $(i)$ it can enclose canal surfaces more closely; (ii) it can be constructed rather easily; (iii) more important, intersections of two BCs can be computed geometrically [4].

\subsection{Hierarchical Construction of Bounding Cylinder Tree}

It is uneasy to construct a smallest $\mathrm{BC}$ for canal surfaces theoretically. In practice, it is acceptable to construct a near $\mathrm{BC}$ for canal surfaces, as long as it can reasonably close to the smallest BC.
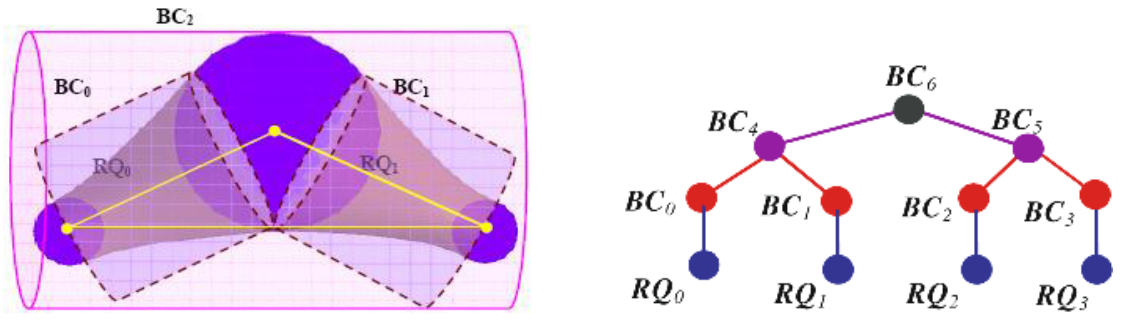

Fig. 2. A binary bounding cylinder tree BCT for a canal surface

Considering that canal surfaces have good geometric properties, they should have simpler but more efficient BC construction methods. We use a hierarchical BC construction method for canal surfaces. In our $R Q$-sphere decomposition, a canal surface is subdivided as a set of $n G^{l} R Q$-spheres. It is easy to construct a bounding cylinder BC for each $R Q$. For two neighboring $R Q_{0}$ and $R Q_{l}$, we can have two bounding cylinders $B C_{0}$ and $B C_{l}$ in a straightforward way, and as shown in Fig. 2, a bigger bounding cylinder $B C_{2}$ can be constructed geometrically to enclose $B C_{1}$ and $B C_{2}$. In the same manner, a binary tree of bounding cylinders HBCT can be organized hierarchically.

\subsection{Canal Valid Intersection Intervals (CVII)}

Suppose that two canal surfaces are subdivided into $n$ and $m R Q$-sphere pairs respectively. A brute force method for canal/canal intersection would require invoking $n * m$ 
$R Q / R Q$ intersection computations. However, it becomes quite inefficient when two canal surfaces only intersect each other within a small overlapping region (see Fig. $3(1)$ ), in this case, only few $R Q / R Q$ pairs of the two canal surfaces within their overlapping regions need to be examined. Therefore, by filtering out those $R Q / R Q$ pairs outside the overlapping regions, the expected performance of the algorithms should improve. To describe it conceptually, canal valid intersection intervals CVII is defined as the interval pairs (u-interval, v-interval) corresponding to the overlapping regions of the two canal surfaces, hereafter denoted by $\left(\mathrm{CVII}_{\mathrm{u}}, \mathrm{CVII}_{\mathrm{v}}\right)$. The idea, bounding cylinders clipping, hereafter denoted by $B C$ clipping, is also proposed to detect $\left(\mathrm{CVII}_{\mathrm{u}}, \mathrm{CVII}_{\mathrm{v}}\right)$, or to find all those potential intersecting $R Q / R Q$ candidates for more efficient intersection determination.

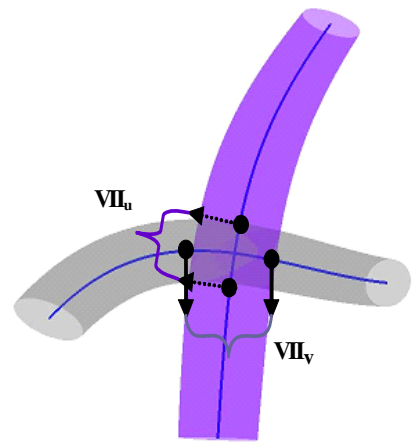

(1)

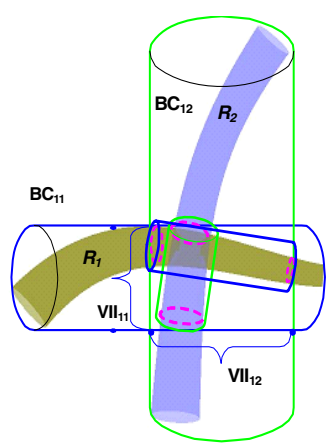

(2)

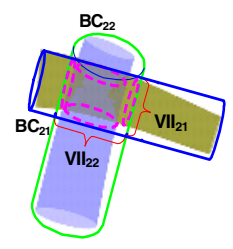

Fig. 3. The valid intersection intervals of two canal surfaces: (1) Definition of VII of two overlapping canal surfaces; (2) Two consecutive rounds of BC clipping

\subsection{Clipping of Two Bounding Cylinders}

It is difficult to determine the exact interval $\left(\mathrm{CVII}_{\mathrm{u}}, \mathrm{CVII}_{\mathrm{v}}\right)$ of two canal surfaces $R_{l}$ and $R_{2}$ directly by solving complicated equations numerically. Therefore, it is acceptable to estimate $\left(\mathrm{CVII}_{\mathrm{u}}, \mathrm{CVII}_{\mathrm{v}}\right)$ of $R_{l}$ and $R_{2}$ only approximately but more efficiently.

Similar to computing RVII of two surfaces of revolution [9, 10], we propose $B C$ clipping to estimate $\left(\mathrm{CVII}_{\mathrm{u}}, \mathrm{CVII}_{\mathrm{v}}\right)$ of two canal surfaces approximately by computing the overlapping regions of their respective bounding cylinders $\mathbf{B C}_{\mathbf{1 1}}$ and $\mathbf{B C}_{\mathbf{1 2}}$, then refining the regions recursively as shown in the right part of Fig. 3(b). The first round of $B C$ clipping for $\mathbf{B C}_{11}$ and $\mathbf{B C}_{\mathbf{1 2}}$ yields the initial intersection interval $\left(\mathrm{CVII}_{11}\right.$, $\mathrm{CVII}_{12}$ ). It is a very rough approximation to the real CVII, within which there are still some $R Q$-sphere pairs of $R_{1}$ and $R_{2}$ that have no intersection, since $\mathbf{B C}_{11}$ and $\mathbf{B C}_{12}$ enclose $R_{1}$ and $R_{2}$ rather loosely. Furthermore, two smaller bounding cylinders $\mathbf{B C}_{\mathbf{2 1}}$ and $\mathbf{B C}_{22}$ are constructed respectively for those $R Q$-sphere pairs within $\mathrm{CVII}_{11}$, and $\mathrm{CVII}_{12}$, then, the second round $B C$ clipping is taken on $\mathbf{B C} \mathbf{C}_{21}$ and $\mathbf{B C} \mathbf{C}_{22}$ as shown in the left part of Fig. 3 (b) (amplified version of second round of $2^{\text {nd }} B C$ clipping), giving a smaller intersection interval pair $\left(\mathrm{CVII}_{21}, \mathrm{CVII}_{22}\right)$. Usually, very few rounds of such $B C$ clipping output very close to the real $\left(\mathrm{CVII}_{\mathrm{u}}, \mathrm{CVII}_{\mathrm{v}}\right)$ of two canal surfaces. 


\section{Computing Intersections of Two Canal Surfaces}

The intersection curve of two canal surfaces, hereafter denoted CSIC, is computed in three steps: ( $i$ ) computing CVII $\left(\mathrm{CVII}_{\mathrm{u}}, \mathrm{CVII}_{\mathrm{v}}\right)$ of the two canal surfaces; (ii) computing all the intersection curve segments of all potential $R Q / R Q$ pairs, RQIC, within $\left(\mathrm{CVII}_{\mathrm{u}}, \mathrm{CVII}_{\mathrm{v}}\right)$ by using Goldman's method [5]; (iii) concatenating all the individual $R Q I C$ into CSIC as a set of independent components (open branches or closed loops).

\subsection{Computing Intersection Curves of Two Canal Surfaces}

The procedure, Find_CVII $\left(\mathrm{BCT}_{1}, \mathrm{BCT}_{2}\right)$, is to compute $\left(\mathrm{CVII}_{11}, \mathrm{CVII}_{12}\right)$ of two canal surfaces. There are three possible cases which should be treated differently:

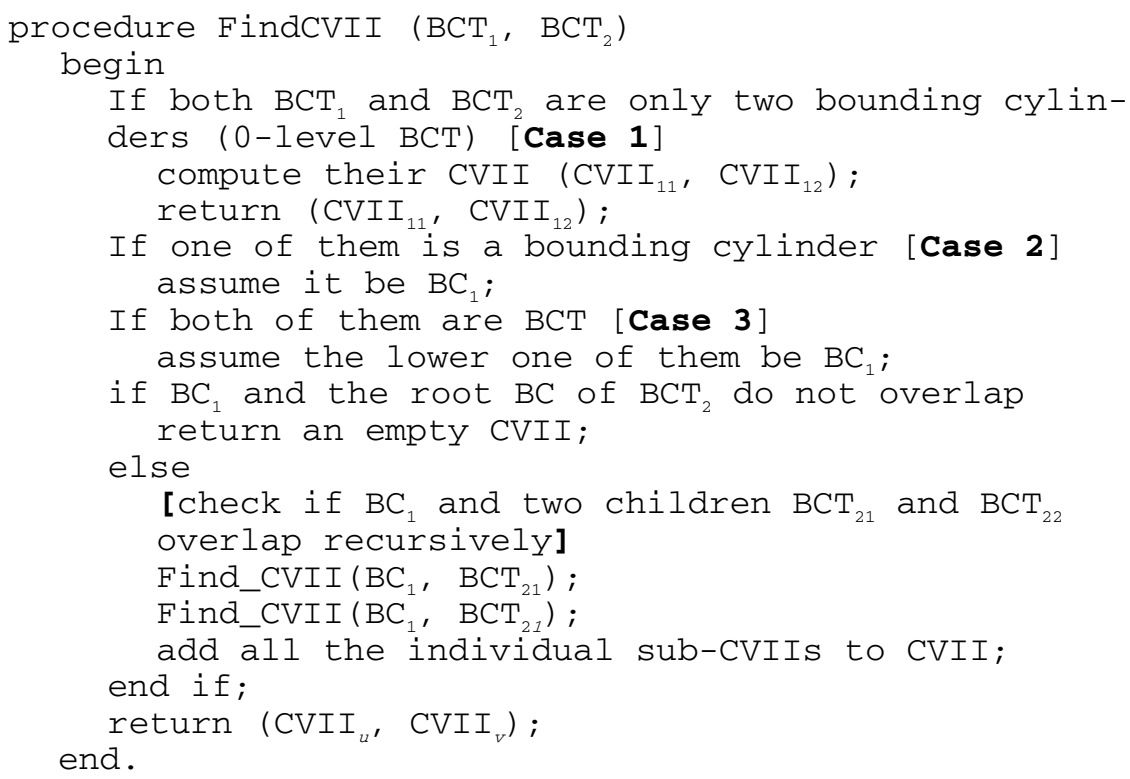

The main idea of our computing intersection curves of two canal surfaces CSIC based on $R Q$-sphere decomposition can be sketched as follow:

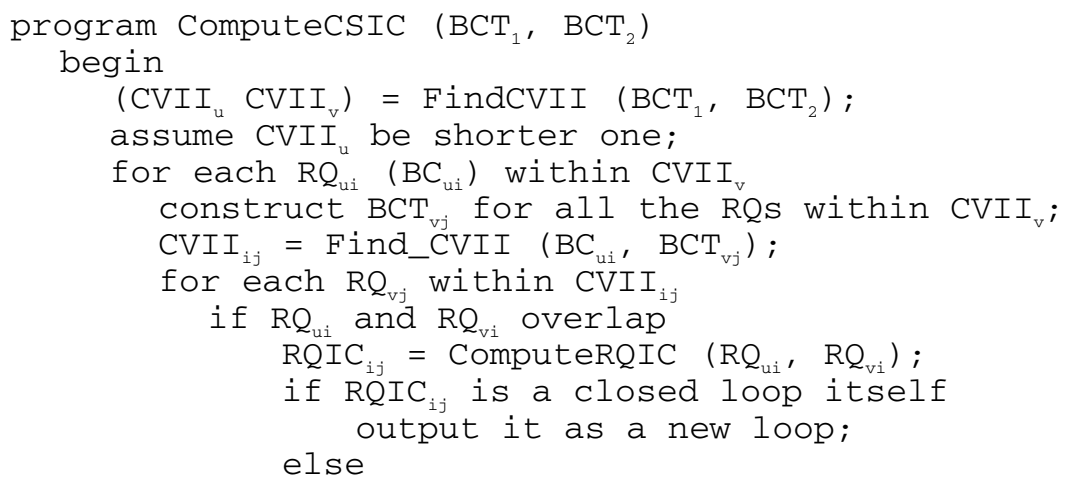




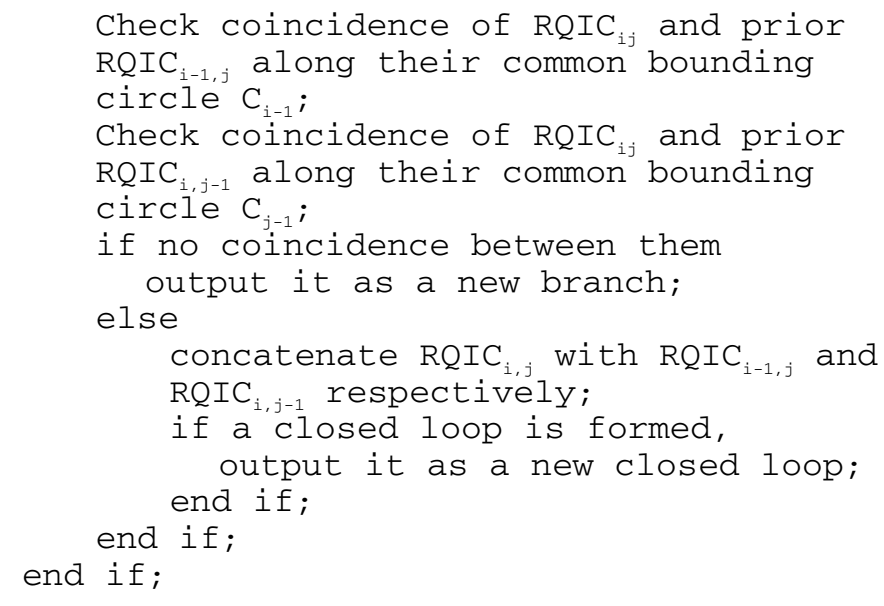

end.

\subsection{Computing Self-intersections of a Single Canal Surface}

Similarly, self-intersection of a single canal surface $R$ can be solved. Assume that the bounding cylinder tree of $R$ is $B C T_{0}, R$ is decomposed $n R Q$-sphere pairs $\operatorname{RQS}_{\mathrm{i}}(i=1$, $2, \ldots, n), B C T_{0}$ has two sub-trees $B C T_{\text {Left }}$ (enclosing the first half $\mathrm{RQS}_{\mathrm{i}}, i=1,2, \ldots$, $[n / 2])$ and $B C T_{\text {Right }}$ (enclosing the other half $\left.\mathrm{RQS}_{\mathrm{i}}, i=[n / 2],[n / 2]+1, \ldots, n\right)$. If $B C T_{\text {Left }}$ and $B C T_{\text {Right }}$ overlap, then, $R$ may intersect itself and its self-intersection curves can be computed by calling $C S I C\left(B C T_{\text {Left }}, B C T_{\text {Right }}\right)$. Otherwise, conquer each half (subtree) recursively. This idea is sketched roughly as follows.

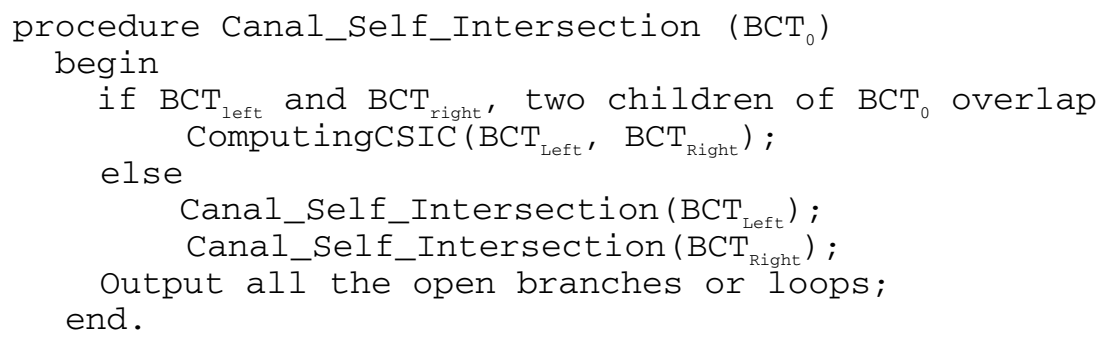

\section{Illustrative Examples}

The proposed algorithms have been implemented with $C++$ and OpenGL under Windows $X P$ and PC (Pentium III, 512M RAM, 512M HZ). Two examples are given in Fig. 4, one is for computing general CSIC, the other is for self-intersection. Their spine curves and radii are represented with cubic Beizer form. Both of them are computed within one second.

Comparing determination method of global self-intersection for a single pipe surface in [16], it is simpler algorithmically, easier for implementation and also more robust, since both fundamental intersection computations of $\mathrm{BC} / \mathrm{BC}$ and $R Q / R Q$ have closed form solutions. 


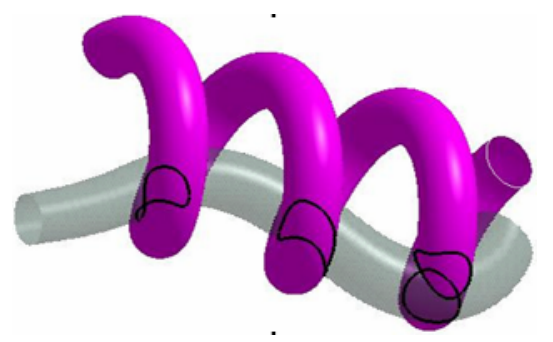

(1)

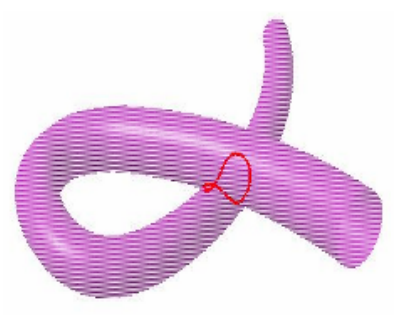

(2)

Fig. 4. Intersection Curves on canal surfaces: (1) The intersection curves of two canal surfaces; (2) Self-intersection curves of a single canal surface

\section{Conclusion}

Robustness is one of most important factors for surface intersection algorithms. We have shown and analyzed the instability of the method [6] in our previous work [9]. Our $R Q$-sphere decomposition based method reduces computing intersections of two canals to computing intersections of two $R Q \mathrm{~s}$, which can be solved by Goldman's method [5] robustly and efficiently. Further, BC clipping makes computing the CSIC relatively efficient. In fact, the efficiency, accuracy and robustness of two revolute quadrics $R Q / R Q$ intersection can be further enhanced by more recent algorithms [1, 2, $18,19]$. Also, $R Q$-sphere decomposition facilitates tracing the intersection curves because it is easy to recognize the closed loops and singular points on the intersection curves. Therefore, both theoretical analysis and practical implementation show the robustness and efficiency of our proposed method.

The $R Q$-sphere decomposition of canal surfaces also can be extended to solving other geometric problems of canal surfaces, e.g. collision detection, isophotes, silhouette, bisector, distance computing and so on.

\section{References}

1. Dupont L., Lazard D., Lazard S., Petitjean S.: Towards The Robust Intersection of Implicit Quadrics. In Proc. of Workshop on Uncertainty in Geometric Computations, Sheffield, UK, (2001).

2. Dupont L., Lazard D., Lazard S., Petitjean S.: Near-Optimal Parameterization of the Intersection of Quadrics. In ACM Symposium on Computational Geometry, San Diego, USA, (2003).

3. Elber G., IRIT Software, http://www.cs.technion.ac.il/ irit/, The Technion-IIT, Haifa, Israel, (1993).

4. Eberly David: Intersection of two cylinders. Magic Software Company. http://www.magic -software. com/ Intersection.html (2000).

5. Goldman R. N.: Quadrics of Revolution. IEEE Computer Graphics and Applications. 3(2), (1983) 68-76.

6. Heo H. S., Hong S. J., Seong J. K., Kim M. S.: The Intersection of Two Ringed Surfaces and Some Related Problems. Graphical Model. 63(4), (2001) 228-244. 
7. Johnstone J.: A New Intersection Algorithm for Cyclides and Swept Surfaces Using Circle Decomposition. CAGD, 10(1). (1993) 1-24.

8. Jia J., Tang K., Joneja A, Kwok K. W.: New Algorithm of Computing Planar Sections of Surfaces of Revolution Based on Revolute Quadric Subdivision”. Proc. of Shape Modeling and Applications. Genova. Italy. (2004).

9. Jia J., Baciu G., Kwok K. W.: Revolute Quadric Decomposition for Computing Intersection Curves of Surfaces of Revolution. Graphical Model. 55(5). (2004) 363-383.

10. Jia J., Tang K, Joneja A.: Bi-Conic Subdivision for Surfaces of Revolution and Its Applications to Intersection Problems. The Visual Computer. 20(7), (2004) 457-478.

11. Jia J., Joneja A. and Tang K., "Revolute Quadric Decomposition of Canal Surfaces and Its Application”. LNCS. Vol. 3514. Springer-Verlag. (2005) 213-222.

12. Kim M. S.: Intersecting Surfaces of Special Types. Proc. of Shape Modeling and Processing. University of Aizu, Japan. (1999) 122-128.

13. Levin J. Z.: A Parametric Algorithm for Drawing Pictures of Solid Objects Composed of Quadrics. Communications of the ACM. 19(10). (1976) 555-563.

14. Levin J. Z..: Mathematical Models for Determining The Intersections of Quadric Surfaces. Computer Graphics and Image processing. Vol. 1, (1979) 73-87.

15. Nelson Max: Cone-Spheres. ACM SIGGRAPH Computer Graphics, Vol. 24, (1990) 59-62.

16. Patrikalakis N. M., Maekawa T.: Shape Interrogation in Computer Aided Design and Manufacturing. Springer Verlag. (2002).

17. Seong J. K., Kim K. J., Kim M. S., Elber G.: Intersecting a Freeform Surface with a Ruled or a Ringed Surface. Proceedings of Geometric Modeling and Processing. Beijing, (2004) 38-45.

18. Wang W., Goldman R., Tu C.: Enhancing Levin's Method for Computing QuadricSurface Intersections. CAGD, 20(7). (2003) 401-422.

19. Wang W., Joe B., Goldman R.: Computing Quadric Surface Intersections Based on An Analysis on Plane Cubic Curves. Graphical Model. 64(6), (2003) 335-367.

20. Paluszny, M., Bühler, K.: Canal Surfaces and Inversive Geometry. Mathematical Methods for Curves and Surfaces II. In Daehlen M., Lyche T., Schumaker L. (eds.), (1998) 367-375. 